\title{
CATTURA, TRATTAMENTO E RIUTILIZZO DELL'ACQUA GRIGIA E PIOVANA NELLA RESIDENZA UNIFAMILIARE
}

\section{RECENSIONE ARTICOLO}

MONTEIRO, Otávio Pinetti ${ }^{1}$

LIMA, Gemael Barbosa ${ }^{2}$

MONTEIRO, Otávio Pinetti. LIMA, Gemael Barbosa. Cattura, trattamento e riutilizzo dell'acqua grigia e piovana nella residenza unifamiliare. Revista Científica Multidisciplinar Núcleo do Conhecimento. Anno 05, Ed. 01, Vol. 01, pp. 98-113. gennaio 2020. ISSN: 2448-0959, Collegamento di accesso: https://www.nucleodoconhecimento.com.br/ambiente/acqua-di-cenere

\section{RIEPILOGO}

L'obiettivo di questo lavoro è quello di presentare una revisione bibliografica, il cui soggetto è il riutilizzo dell'acqua sotto la pioggia, nonché il riutilizzo delle acque grigie in una residenza unifamiliare. Per questo, la metodologia costituisce una ricerca di informazioni in libri, riviste, articoli, tesi di laurea magistrale e tesi di dottorato. Per riutilizzare consapevolmente, modi per catturare l'acqua da una residenza, sia dalla pioggia che cade sulle grondaie o utilizzato nei metodi di cattura nei lavandini da cucina e / o serbatoi di aree di servizio che riceveranno il trattamento necessario e saranno riutilizzati. Questi trattamenti agiscono in modo efficiente e forniscono l'uso di questa

${ }_{1}$ Post-laurea in Ingegneria Ambientale e Sicurezza Occupazionale (Faveni), Postlaurea in Sostenibilità nell'Ambiente Costruito (Ifes), Post-Laureato in Insegnamento dell'Istruzione Superiore (Faveni), Laureato in Ingegneria Civile (Unesc), Laureato in Matematica.

${ }^{2}$ Laurea magistrale in Ingegneria Ambientale, professore presso I'UNESC. 
acqua per scopi meno nobili che rendono il consumo più razionale ed economico, cooperando per il mantenimento delle risorse ambientali.

Parole chiave: acqua, riutilizzo, cattura, trattamento, residenza.

\section{INTRODUZIONE}

Come afferma il 2005, l'acqua è diventata un simbolo comune dell'umanità, valorizzata in tutte le religioni e culture, anche un simbolo di uguaglianza sociale, perché la crisi idrica è soprattutto la distribuzione, le risorse e non solo nella sua assoluta scarsità . Con il chiaro obiettivo di ridurre il consumo di acqua, attraverso nuove tecnologie, è iniziato lo sviluppo di diversi programmi di uso razionale.

Questi problemi associati confermano la discussione sulla fattibilità di fare l'uso di acqua piovana e acqua grigia per le attività fondamentali in una residenza. Questa possibilità di riutilizzo comporterà certamente la diminuzione del consumo di acqua trattata fornita dalle aziende igienico-sanitarie, inoltre, ci sarà una diminuzione della domanda di costi con l'uso di acqua potabile e la riduzione dei rischi derivanti da inondazioni in periodi di forti piogge.

Pertanto, la ricerca affrontata ha lo scopo di dimostrare come il riutilizzo dell'acqua dalla pioggia viene fatto insieme al riutilizzo dell'acqua grigia in una residenza unifamiliare, dalla presentazione di un caso di studio. L'intera base del lavoro è stata trovata attraverso informazioni in libri, riviste, articoli e tesi di laurea, in cui gli autori e i ricercatori del settore, della ricerca di laboratorio e accademica sono stati utilizzati per sostenere i loro risultati.

Sebbene l'acqua sia una risorsa rinnovabile, la sua offerta non soddisfa la domanda che nel tempo è aumentata in modo sostanziale. Con questo ci sarà sempre un deficit, sempre più accentuato da un uso irrazionale e da vari rifiuti. Pertanto, è sempre più necessario stabilire un nuovo rapporto tra l'uomo e l'acqua, poiché questo viene ampliato le opzioni di uso razionale e aggiungendo a questo fattore, alternative per trarre vantaggio in modo efficiente, sia attraverso la cattura e il trattamento di come 
avvicinato sul lavoro o con metodi più specifici che non sono ancora ben accolti dalla popolazione per avere il prezzo di investimento molto alto.

\section{QUADRO TEORICO}

[...] In combinazione con la capacità di dissoluzione di cui sopra, l'acqua agisce come mezzo di trasporto - in superficie e in clandestinità permettendo alle caratteristiche dello stesso corso d'acqua di cambiare temporalmente e spazialemente. (LEBANON, 2010, p.15)

La potabile delle acque naturali afini di consumo umano ha come funzione essenziale adattare l'acqua grezza ai limiti fisici, chimici, biologici e radioattivi stabiliti dall'ordinanza 2914, rendendo gli effluenti della stazione incapaci di trasmettere dannoso per la popolazione fornita.

[...] II trattamento dell'acqua consiste nell'eliminazione di particelle sospese e colloidali, materia organica, microrganismi e altre sostanze deleterie per la salute umana, forse presenti nelle acque naturali, ai costi più bassi di impianto, funzionamento e manutenzione, e generando il minor impatto ambientale sulle aree circostanti. (LIBÂNIO, 2010, p.135).

\subsection{USO DELL'ACQUA NELL'AMBITO DELLA COSTRUZIONE CIVILE}

Secondo Mayok (2009), ci sono altri standard, come NBR 7229/1993, che ha le dimensioni del sistema idrico servito; e le linee guida per la conservazione e il riutilizzo dell'acqua negli edifici preparati dall'Unione delle Costruzioni Civili (Sinduscon), che presenta diversi modi per riutilizzare l'acqua, anche nel lavaggio degli aggregati per I'uso nell'edilizia civile.

Secondo Sickermann (2005) citato da PROSAB, 2006 come una risorsa estremamente importante per la costruzione, l'acqua viene utilizzata in tutte le fasi di un lavoro, dalla produzione di calcestruzzo anche per soddisfare le esigenze di base dei lavoratori - e la sua scarsità è già avvertita in cantieri in diverse località del paese. 
Secondo il vice presidente dell'Unione delle costruzioni (Sinduscon-MG)Geraldo Jardim Linhares Jànio, anche se la situazione non è ancora grave, le imprese di costruzione hanno optato per un po 'di tempo per sostituire i metodi convenzionali con metodi di costruzione a secco, al fine di creare una certa indipendenza dall'acqua (MARIANE, 2014).

\subsection{USI MULTIPLI DI ACQUA}

Sulla base della ricerca di Tomaz (2005) per quanto riguarda il riutilizzo dell'acqua piovana, l'uso di misure e tecniche è eccellente per evitare e porre rimedio ai problemi circa la mancanza di acqua potabile, anche senza leggi che ne attuino l'uso. L'uso di metodi appropriati e l'uso alternato con l'acqua proveniente dalle fogne domestiche sarebbero un modo intelligente per conservare l'acqua per scopi nobili.

Come indicato da Caubet (2006), rappresentante delle organizzazioni non governative (ONG) nel Consiglio nazionale per le risorse idriche $(\mathrm{CNRH})$, anche se il codice dell'acqua (decreto federale n. 24.643, dal 10/07/1934) presenta la priorità assoluta dell'uso dell'acqua per i bisogni fondamentali dell'uomo (desizione e uso domestico), il più grande ritiro avviene nel settore agricolo $(62,7 \%)$, poi avviene attraverso il consumo umano $(17,9 \%)$ e l'uso industriale (14\%) e, infine, nel consumo di animali $(5,4 \%)$, come illustrato nella figura 01.

Figura 01 -Consumo di acqua per attività in Brasile.

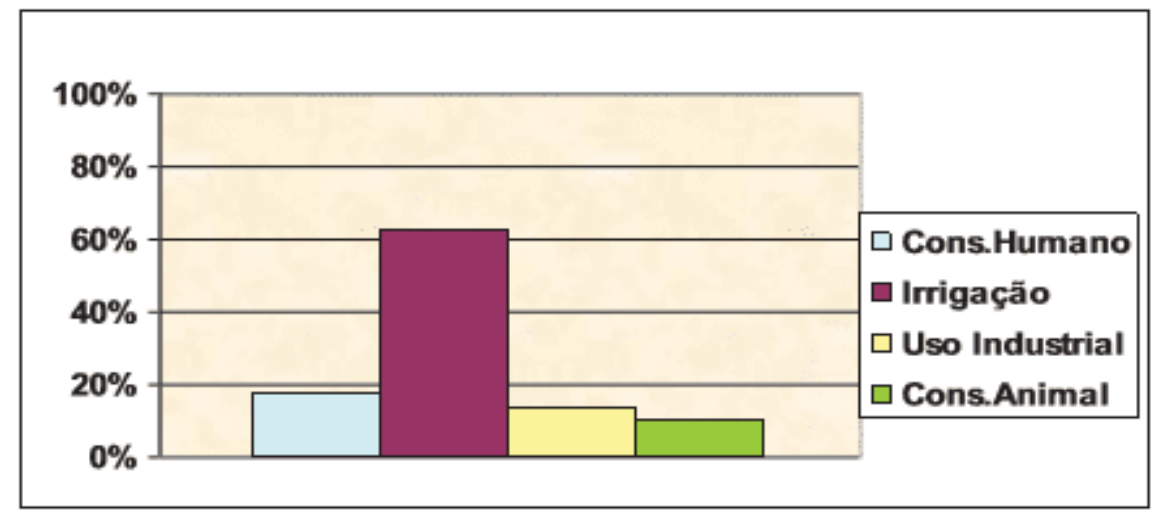

Fonte: ANA (2002) citata da MANUAL FIESP/CIESP (2006). 


\subsubsection{USI DI ACQUA NON POTABILE}

Studi condotti in Brasile e all'estero mostrano che in una residenza, gran parte del consumo di acqua si concentra sui bagni, lo scarico di servizi igienici, lavandino della cucina e lavare i vestiti. In media, il $40 \%$ dell'acqua totale consumata in una residenza è destinata all'uso in modo non potabile. (GONÇALVES, 2009).

La necessità di analizzare la quantità di acqua distribuita per soddisfare le attività di base di consumo e igiene è motivo di discussione tra diversi autori, come possiamo analizzare dalla Figura 02. Una definizione proposta da Peter Gleick, contenuta nel Basic Water Requirement (BWR), è che $50 \mathrm{~L} /$ hab/giorno sono una quantità sufficiente per soddisfare queste esigenze (BIO, 2002).

Figura 02 - Distribuzione del consumo idrico nelle case da parte di ciascun apparecchio

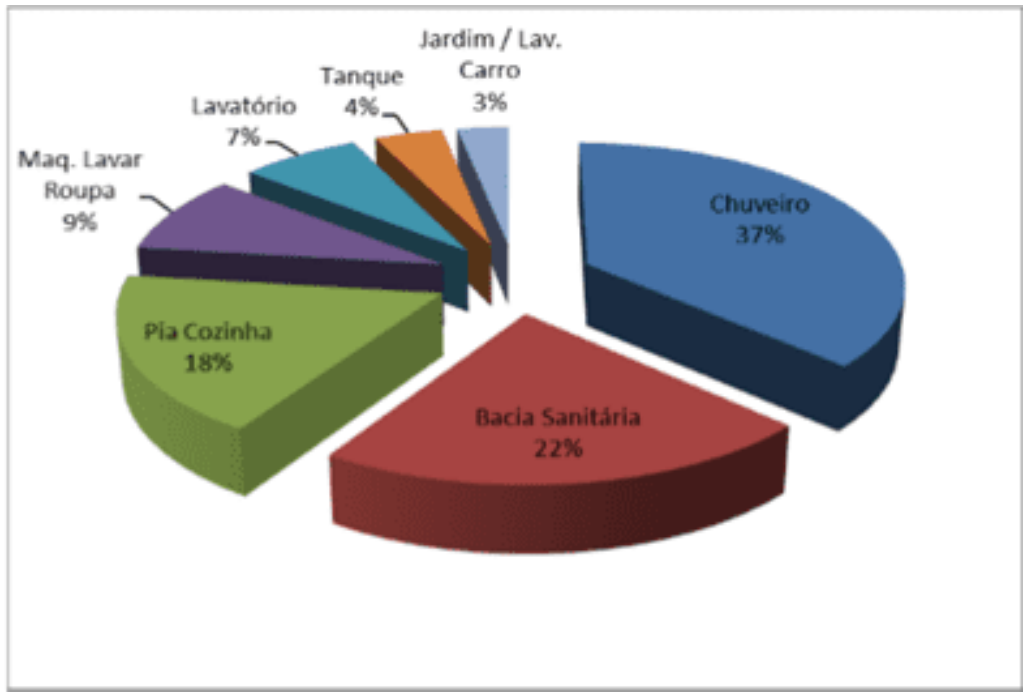

Fonte: Adattato da Martins e Memelli (2011)

\subsubsection{USI DI ACQUA POTABILE}

L'acqua potabile è tutta acqua adatta al consumo. Essendo un liquido incolore, inodore, insapore e disossato, essenziale per la sopravvivenza umana, come afferma Hespanhol (2002). 


\subsubsection{TRATTAMENTO}

Non è essenziale che l'acqua in primavera presenti tutti gli indicatori appropriati per il consumo, perché secondo Braga (2005) ci sono modi per modificarne le caratteristiche per renderla compatibile con le esigenze di salute pubblica.

Sempre secondo Braga (2005), i principali metodi di trattamento delle acque sono: decantazione, flocculazione, filtrazione, disinfezione, rimozione della durezza, aerazione, rimozione di ferro e manganese, rimozione di sapore e odore, controllo della corrosione e fluorizzazione, che sono sempre interconnessi e che la mancanza di un trattamento adeguato di alcuni di questi elementi può alterare la qualità finale dell'acqua.

\section{MATERIALI E METODI}

La metodologia di questo articolo consisteva nella ricerca bibliografica in libri, riviste scientifiche, tesi di dottorato e tesi di laurea magistrale. La revisione si è limitata ai modi per catturare e sfruttare l'acqua piovana, inoltre, è stato discusso anche sull'uso dell'acqua grigia. L'obiettivo di questo lavoro era quello di occuparsi delle tecniche di cui sopra nel contesto della residenza unifamiliare.

Per basare meglio la revisione bibliografica proposta in questo articolo, è stato presentato un caso di studio di Oliveira (2005) nel comune di Palhoa, situato a $15 \mathrm{~km}$ da Florian-polis, nello stato di Santa Catarina. Dopo aver presentato i risultati della ricerca di oliveira (2005), la discussione è stata condotta portando altri studi simili.

Nelle sezioni seguenti saranno presentati l'area di studio e l'obiettivo, nonché le caratteristiche della residenza, tra gli altri aspetti del caso di studio scelto per citare questo articolo.

\subsection{AREA DI STUDIO}

II comune scelto per l'attuazione del progetto è stato Palhoa, situato a $15 \mathrm{~km}$ da Florian-polis tra la costa e la Serra do Mar, nel meridiano 48040'04" longitudine ovest 
e parallelo 280 38' 43' latitudine sud. Ha una superficie di $323 \mathrm{~km} 2$, di cui il $73,0 \%$ del territorio è considerato area di conservazione permanente. La popolazione del comune, secondo IBGE (2004), è di 113.312 abitanti, secondo (PALHOA, 2004). Secondo Setti (1994) si trova a tre metri sul livello del mare, su una pianura balneare con mangrovie, riposo e massicci rocciosi della Serra do Mar. II clima, secondo l'autore, è umido con una temperatura media di $250 \mathrm{C}$. Le precipitazioni medie annue dal 1969 al 2002, secondo CLIMERH/EPAGRI è di 1706 mm.

\subsection{OGGETTO DI STUDIO}

Lo studio si basava su due residenze distinte e si trovava ad una distanza di $2 \mathrm{~km}$ l'una dall'altra. Residence 01 ha una superficie di $131.36 \mathrm{~m} 2$ e la residenza 02 ha una superficie di $143.27 \mathrm{~m} 2$. Nella residenza 01, i punti di consumo si trovano nel bagno, nell'area di servizio e nella cucina. II bagno è costituito da una doccia, un lavabo, un bagno con scarico valvola. In cucina, c'è un rubinetto sul lavandino. Nell'area di servizio, c'è una lavatrice che utilizza 100 litri di acqua per ciclo e un rubinetto situato nel serbatoio. Nella residenza 02, i punti di consumo dell'acqua sono situati in tre bagni, cucina e area di servizio. Nei tre bagni ci sono due docce, tre rubinetti per lavabo, due con miscelatori e tre scarichi sanitari. In cucina, c'è un rubinetto con mixer e nell'area di servizio c'è una lavatrice con consumo di 80 l per ciclo e un rubinetto nel serbatoio. In entrambe le residenze praticamente nessuna acqua potabile viene utilizzata per lavare marciapiedi, automobili o irrigazione da giardino. L'uso dell'acqua utilizzata dalla lavatrice avviene già in entrambe le residenze, conservandola in serbatoi per un successivo riutilizzo su marciapiedi e giardini.

\subsection{SISTEMA DI RACCOLTA E STOCCAGGIO DELL'ACQUA PIOVANA E RESO}

\subsubsection{DETERMINAZIONE DELLE AREE DI COPERTURA}

Per immagazzinare l'acqua proveniente dalla pioggia è necessario sorvegliare le aree del tetto di entrambe le residenze. La determinazione di queste aree è stata effettuata 
attraverso una vasta analisi dei piani di copertura delle residenze proposte che sono gli stessi importanti nella cattura.

\subsubsection{COLLEZIONE DI ACQUA TETTO}

L'acqua piovana viene catturata dalle grondaie e portata al serbatoio inferiore attraverso conduttori verticali, scalata secondo NBR 10844 (ABNT, 1989). Questo standard riguarda le installazioni di costruzione di acqua piovana.

\subsubsection{SERBATOIO PER LA CATTURA DELL'ACQUA PIOVANA}

Al fine di raggiungere il volume del serbatoio che immagazzina l'acqua dalla pioggia, sono state analizzate le aree contributive della copertura delle case, il consumo giornaliero di acqua per abitante, nelle precipitazioni della regione e nel coefficiente di utilizzo dell'acqua. Pioggia. Questo coefficiente mostra la percentuale che viene immagazzinata, perché il resto viene utilizzato per la pulizia del tetto, grondaie e tubi ed evaporazione. Per questo studio, sarà adottato che l' $80 \%$ dell'acqua che cade sul tetto sarà raccolto.

In ogni residenza ci saranno due serbatoi, uno inferiore che renderà lo stoccaggio dell'acqua piovana raccolta e quello superiore che verrà utilizzato per distribuire le acque per il consumo. II fondo sarà in fibra di vetro sepolto nel terreno. Lo smaltimento della prima acqua piovana sarà effettuato lungo il tubo situato prima che l'acqua entri nel serbatoio. La prima pioggia è tenuta in questo tubo, impedendo alla maggior parte delle impurità di raggiungere il serbatoio. Per la ritenzione di rifiuti più grandi sarà installato presso l'uscita delle grondaie una griglia pvc flessibile. Alla fine del tubo di inseriazione dell'acqua nel serbatoio, verrà installata una riduzione e un ginocchio di 90 gradi con un diametro maggiore del tubo di inseridio. L'aumento dell'area riduce l'impatto dell'acqua piovana raccolta dal fondo del serbatoio, evitando così l'agitazione delle particelle depositate (Figura 03). 
Figura 03 - Modello di riserva utilizzato nel sistema di acquisizione e archiviazione.

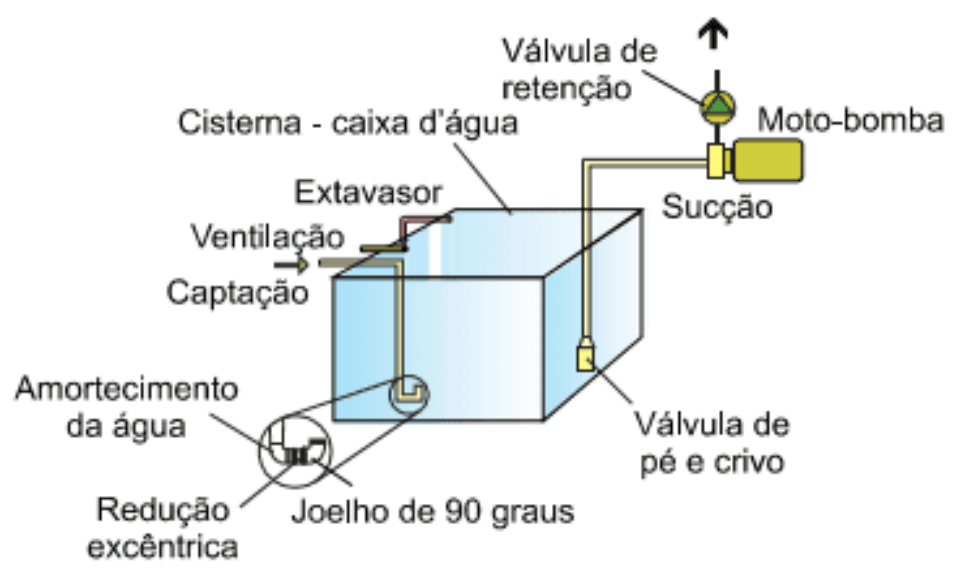

Fonte: ANA (2002) citata da MANUAL FIESP/CIESP (2006).

II serbatoio superiore sarà alimentato attraverso un sistema di pompaggio del motore. Tra il serbatoio superiore di acqua piovana e il serbatoio di acqua potabile ci dovrebbe essere un sistema "by pass" che fornirà la mancanza di acqua piovana con acqua potabile.

\subsubsection{SERBATOIO PER IL RIUTILIZZO DELL'ACQUA}

I serbatoi per il riutilizzo avranno volumi legati al volume di acque reflue secondarie (acqua di bagno, lavabo, lavatrice e acqua rasata) generato in entrambe le residenze. Questi volumi saranno verificati attraverso un'indagine effettuata presso il sito di consumo idrico. In ogni residenza ci saranno due serbatoi: un impiantato più basso per lo stoccaggio dell'acqua e un superiore solo per la distribuzione del consumo. II serbatoio inferiore sarà in fibra di vetro e sepolto nel terreno. Prima di raggiungere il serbatoio, le acque reflue secondarie subiranno una scatola di rimozione della sabbia per la conservazione dei suoi rifiuti e un sistema di trattamento delle piante chiamato zona radice. Dopo aver immagazzinato l'acqua già con un trattamento adeguato, sarà guidato al serbatoio superiore attraverso un sistema di pompa moto e quindi alimentare i punti di utilizzo. 


\section{RIISULTATI}

Per il progetto presentato da Oliveira (2005) in cui viene effettuata la cattura, lo stoccaggio e la distribuzione dell'acqua piovana e del riutilizzo, è stata effettuata un'indagine sugli usi finali di questa acqua nel comune di Palhoa. Dalla determinazione di questi usi finali, si può determinare il volume di acqua necessaria per l'uso dell'acqua piovana e il volume delle acque reflue secondarie disponibili per il suo riutilizzo. Dopo aver determinato questi volumi, i sistemi vengono scalati per l'acqua piovana e per il riutilizzo dell'acqua grigia, quindi viene eseguita l'analisi economica per determinare la fattibilità dei sistemi impiantati.

\subsection{PUNTI DI MAGGIORE CONSUMO IN RESIDENZA 01}

Dopo la correzione effettuata attraverso i metodi di sensibilità, il luogo in cui il consumo di acqua più elevato è stata la doccia con il 32,8\% del consumo totale della residenza, seguita dalla toilette con il $30,4 \%$ e dal rubinetto della cucina con il $28,0 \%$, secondo la tabella 01.

Tabella 01 - Descrizione del consumo idrico nei punti di residenza 01

\begin{tabular}{|c|c|c|c|c|c|}
\hline \multirow{2}{*}{ Aparelho } & \multicolumn{4}{|c|}{ Consumo (litros) } & \multirow[b]{2}{*}{$\%$} \\
\hline & $\mathrm{H}_{1}$ & $\mathbf{M}$ & $\mathbf{H}_{2}$ & Total & \\
\hline Chuveiro & 1528,9 & 1662,6 & 2092,8 & 5284,3 & 32,8 \\
\hline Lavatório & 92,8 & 132,1 & 73,8 & 298,7 & 1,9 \\
\hline Vaso Sanitário & 1465,1 & 2289,6 & 1138,6 & 4893,2 & 30,4 \\
\hline Barba & 75,6 & - & 55,2 & 130,8 & 0,8 \\
\hline Máquina Lavar Roupa & 333,3 & 333,3 & 333,3 & 1000,0 & 6,2 \\
\hline Torneira Cozinha & 1505,0 & 1505,0 & 1505,0 & 4515,0 & 28,0 \\
\hline TOTAL & & & & 16122,0 & 100,0 \\
\hline
\end{tabular}

Fonte: Oliveira, 2005

\subsection{PUNTI DI MAGGIORE CONSUMO IN RESIDENZA 02}

Nella residenza 02, dopo la correzione, il consumo più elevato è stato osservato anche nella doccia, con il $45,6 \%$ del consumo totale della residenza, seguito dalla toilette con il $25,6 \%$ e dal lavandino della cucina con il 13,5\%, come indicato nella tabella 02 . 
Tabella 02 - Descrizione del consumo idrico nei punti di residenza 02

\begin{tabular}{lcccc}
\hline \multirow{2}{*}{ Aparelho } & \multicolumn{3}{c}{ Consumo (litros) } & \\
\cline { 2 - 4 } & $\mathbf{M}$ & $\mathbf{H}$ & Total & $\%$ \\
\hline Chuveiro & 1339,2 & 1762,8 & 3102,0 & 45,6 \\
Lavatório & 112,0 & 66,2 & 178,3 & 2,6 \\
Vaso Sanitário & 986,7 & 755,5 & 1742,2 & 25,6 \\
Barba & - & 300,0 & 300,0 & 4,4 \\
Máquina Lavar Roupa & 280,0 & 280,0 & 560,0 & 8,2 \\
Torneira Cozinha & 460,8 & 460,8 & 921,6 & 13,5 \\
\hline TOTAL & & & $\mathbf{6 8 0 4 , 0}$ & $\mathbf{1 0 0 , 0}$ \\
\hline
\end{tabular}

Fonte: Oliveira, 2005

\subsection{USI FINALI PER L'ACQUA PIOVANA E L'ACQUA}

Per determinare il volume giornaliero disponibile per il riutilizzo dell'acqua e il volume giornaliero necessario per l'uso di acqua piovana si ottiene attraverso gli usi finali di acqua dopo la correzione eseguita nel consumo stimato. Le tabelle 03 e 04 osservano la quantità di acqua utilizzata per il riutilizzo, nonché la quantità necessaria per l'approvvigionamento attraverso il riutilizzo e il volume necessario per l'uso dell'acqua piovana. La quantità necessaria di acqua disponibile per l'uso come riutilizzo è stata ottenuta dal consumo nella doccia, lavabo, acqua utilizzata per la rasatura e in lavatrice, per un totale, per la residenza 01, circa 240 litri, che equivale al $41,9 \%$ del consumo residenza totale. Con l'acqua di riutilizzo è possibile fornire solo la toilette, con un bisogno giornaliero di 175,0 litri, circa il 30\% del consumo della residenza. Pertanto, è disponibile acqua sufficiente per fornire il bagno. Utilizzando l'acqua piovana, è possibile fornire la toilette e la lavatrice. Nella residenza 01, vi è un bisogno giornaliero di 210,5 litri, circa il $36,6 \%$ del consumo totale della residenza. 
Tabella 03 - Consumo per il riutilizzo e l'uso dell'acqua piovana in residenza 01.

\begin{tabular}{lccc|ccc|ccc}
\hline \multirow{4}{*}{ Aparelhos } & \multicolumn{10}{c}{ Consumo } \\
\cline { 2 - 10 } & \multicolumn{2}{c|}{ Reúso- Disponível } & \multicolumn{2}{c|}{ Reúso- Necessário } & \multicolumn{3}{c}{ Água Chuva } \\
\cline { 2 - 10 } & $\begin{array}{l}\text { Diário } \\
\text { (litros) }\end{array}$ & $\begin{array}{c}\text { Mensal } \\
\text { (litros) }\end{array}$ & $\%$ & $\begin{array}{l}\text { Diário } \\
\text { (litros) }\end{array}$ & $\begin{array}{c}\text { Mensal } \\
\text { (litros) }\end{array}$ & $\%$ & $\begin{array}{l}\text { Diário } \\
\text { (litros) }\end{array}$ & $\begin{array}{c}\text { Mensal } \\
\text { (litros) }\end{array}$ & $\%$ \\
\hline Chuveiro & 188,7 & 5284,3 & 32,8 & - & - & - & - & - & - \\
Lavatório & 10,7 & 298,7 & 1,9 & - & - & - & - & - & - \\
Vaso Sanitário & - & - & - & 174,8 & 4893,3 & 30,4 & 174,8 & 4893,3 & 30,4 \\
Barba & 4,7 & 130,8 & 0,8 & - & - & - & - & - & - \\
Máquina Lavar Roupa & 35,7 & 1000,0 & 6,2 & - & - & - & 35,7 & 1000,0 & 6,2 \\
\hline TOTAL & $\mathbf{2 3 9 , 8}$ & $\mathbf{6 7 1 3 , 8}$ & $\mathbf{4 1 , 9}$ & $\mathbf{1 7 4 , 8}$ & $\mathbf{4 8 9 3 , 3}$ & $\mathbf{3 0 , 4}$ & $\mathbf{2 1 0 , 5}$ & $\mathbf{5 8 9 3 , 3}$ & $\mathbf{3 6 , 6}$ \\
\hline
\end{tabular}

Fonte: Oliveira, 2005

Tabella 04 - Consumo per il riutilizzo e l'uso dell'acqua piovana in residenza 02.

Fonte: Oliveira, 2005

\subsection{RISPARMIO FINALE CON IL CAPTURE, STORAGE E TREATMENTDIRAGGIO DI RAINWATER E RE-SO}

La tabella 05 mostra i risparmi con l'acqua potabile, riferendosi all'uso del sistema di utilizzo dell'acqua a partire dalla pioggia, all'implementazione del sistema di riutilizzo dell'acqua e all'attuazione dell'acqua piovana e riutilizzo insieme, nelle case 01 e 02 , in valuta ( $\mathrm{e}$ in percentuale.

In residenza 01 , vi è un risparmio annuo con acqua potabile del $35,5 \%$ a causa dell'attuazione del sistema di utilizzo dell'acqua piovana e di circa il $30,4 \%$ con l'uso del sistema di riutilizzo. L'attuazione dell'assemblaggio e dell'acqua piovana ha prodotto risparmi con acqua potabile del $36,4 \%$.

Per la residenza 02, attuazione di un sistema di utilizzo dell'acqua piovana, è stata ottenuta un'economia con acqua potabile del 33,6\%, e con l'attuazione del riutilizzo, $25,6 \%$. L'economia totale con entrambe le forme di cattura è stata del $33,8 \%$. 
Tabella 05 - Risparmiannuali nelle residenze 1 e 2.

\begin{tabular}{lcccc}
\hline \multirow{2}{*}{\multicolumn{1}{c}{ Economia }} & \multicolumn{4}{c}{ Economia } \\
\cline { 2 - 5 } & \multicolumn{2}{c}{ Residência 1 } & \multicolumn{2}{c}{ Residência 2 } \\
\cline { 2 - 5 } & $\mathbf{R S}$ & \% & R\$ & \% \\
\hline Economia anual - água de chuva & 117,10 & 35,5 & 46,75 & 33,6 \\
Economia anual - reúso de água & 100,05 & 30,4 & 35,60 & 25,6 \\
Economia anual - água de chuva + reúso & 119,84 & 36,4 & 47,01 & 33,8 \\
\hline
\end{tabular}

Fonte: Oliveira,2005

\section{DISCUSSIONE}

Per il progetto presentato nella città di Palhoa trattato in questo articolo, il sistema ha utilizzato componenti semplici che soddisfacevano tutte le esigenze di base e che oltre ad essere un metodo semplice, può generare risparmi per i residenti in cui l'investimento iniziale era basso e il ritorno è accaduto in breve tempo. La ricerca mette inoltre a confronto lo stesso metodo applicato in due unità diverse, che mira a stabilire i metodi di costruzione e il loro contributo alla raccolta e allo stoccaggio dell'acqua per il riutilizzo.

Altri autori stabiliscono diversi sistemi per la cattura e il trattamento dell'acqua grigia e piovana, dove l'economia varia a seconda degli investimenti stabiliti da ciascun modello e anche in base all'efficienza del sistema.

Secondo il lavoro di Silveira (2008), la "Europe Line", basata su un'indagine tedesca sul trattamento dell'acqua piovana, comprende sistemi che forniranno un uso domestico ed esterno, servendo anche aree di maggiore cattura. È ideale per le residenze che sono ancora in fase di costruzione, in quanto consente una maggiore integrazione tra i sistemi di bere e acqua piovana. Questo metodo secondo il ricercatore utilizzerà una pompa, oltre ad altri accessori come il freno ad acqua (per ridurre il vortice nella cisterna), filtro galleggiante per consentire una maggiore qualità dell'acqua catturata e multi-sifone in modo da non lasciare che gli insetti entrare nel sistema. La quotatura del serbatoio per lo stoccaggio sarà la stessa di quella trattata nel lavoro svolto nella città di Palhoa, dove è definita attraverso la previsione di consumo, la base di cattura e il tempo asciutto per il sito. Può anche collegare il 
sistema di acqua piovana con l'acqua della rete di approvvigionamento pubblico in modo che nei momenti di mancanza di acqua l'integrazione tra i due sistemi garantisca qualità e approvvigionamento, secondo la figura 04.

Figura 04: Modello della linea Europa affrontato dalla mestranda Bruna Quick da Silveira
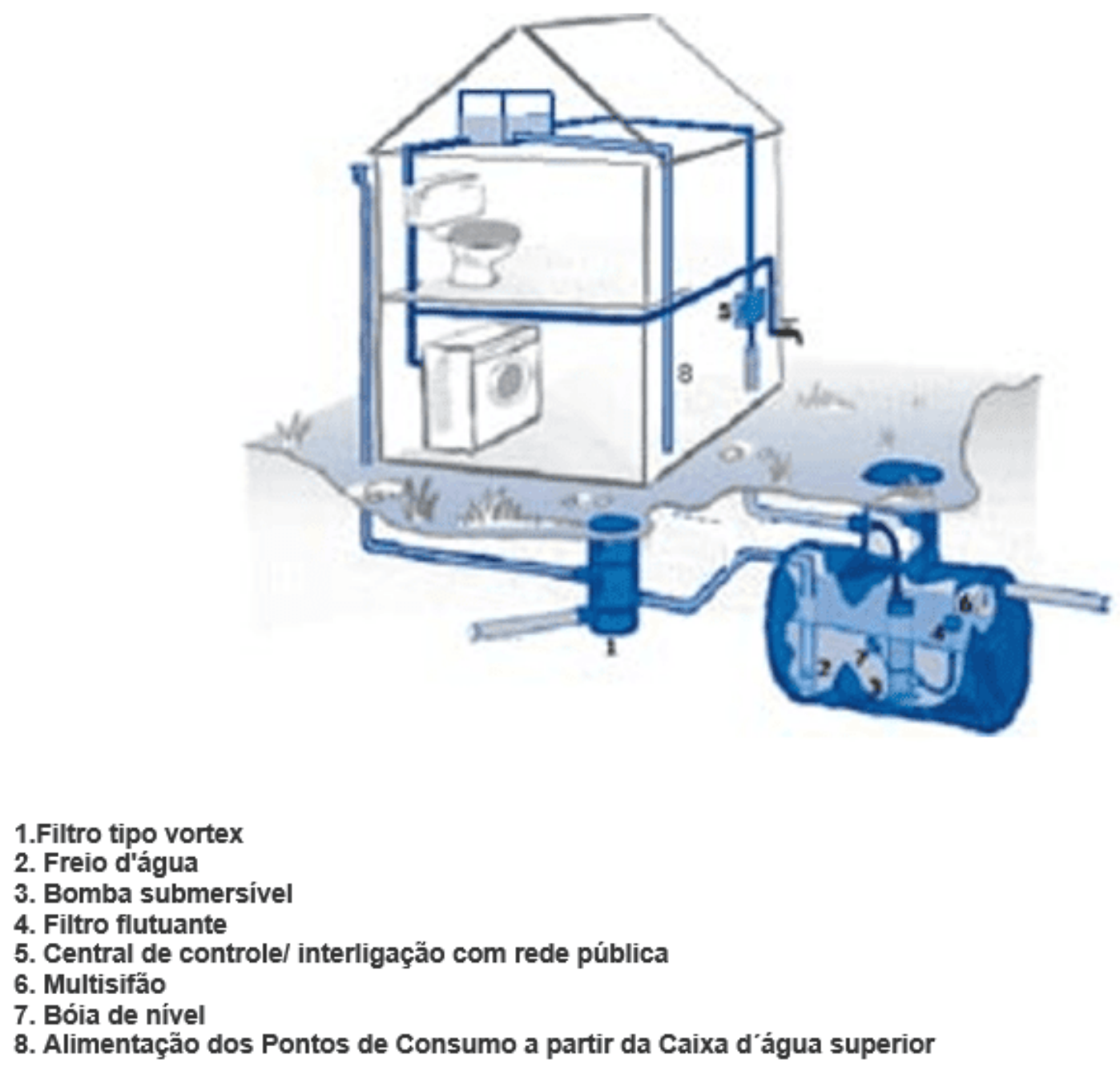

Fonte: AQUASTOCK, 2008.

I due sistemi di fronte sono efficienti, ma utilizzano modi diversi per catturare, trattare e distribuire l'acqua raccolta, dove ogni metodo ha la sua particolarità. L'efficienza di entrambi è soddisfacente che rende i due regimi usuali ed economici, avendo come fattore determinante scegliere l'investimento iniziale più appropriato che ciascuno 
richiederà, essendo quello utilizzato nella città di Palhoa il metodo più semplice, che termina finisce che richiedono un investimento iniziale inferiore a quello presentato dalla mestranda, denominato sistema "Europe Line".

\section{CONCLUSIONE}

Dall'obiettivo di questo articolo, si può percepire che l'interesse della società è piccolo quando l'argomento all'ordine del giorno è l'installazione di un sistema in grado di riutilizzare l'acqua che è stata precedentemente scartata. Ciò è dovuto al fatto che vi è un certo pregiudizio con il riutilizzo dell'acqua proveniente dagli effluenti domestici e anche a causa del maggiore rischio di contaminazione associato al sistema di manutenzione che può promuovere danni alla salute degli utenti diretti o indiretti del sistema. Un metodo di riutilizzo appropriato e gestito consapevolmente aumenta l'efficienza e riduce qualsiasi tipo di contaminazione che può verificarsi da parte dell'operatore o del sistema nel suo complesso.

II modello di cattura presentato in questo lavoro o in qualsiasi altro disponibile al mondo richiede un costo di installazione iniziale molto elevato, ma a lungo termine deve avere risparmi nel volume di acqua utilizzata nella residenza, così come nella qualità dell'acqua che viene ricevuta, perché nel trattamento e nel riutilizzo dell'acqua che una volta restituita alla natura ci sarà la conservazione della qualità di questo bene che, oltre al finito, è essenziale per il benessere e per la qualità della vita umana.

\section{RIFERIMENTI}

ABNT - Associação Brasileira de Normas Técnicas. NBR 10844 - Instalações Prediais de Águas Pluviais. Rio de Janeiro (1989).

ABNT Associação Brasileira de Normas Técnicas. NBR 5626 - Instalações Prediais de Água Fria. Rio de Janeiro (1998).

ANA - Agência Nacional de Águas. A Evolução da Gestão dos Recursos Hídricos no Brasil / The Evolution of Water Resources Management in Brazil. Brasília; ANA, 2002. 
AQUASTOCK - Água da Chuva. Sistema de Reaproveitamento da Água da Chuva. Disponível em: <http://www.engeplasonline.com.br> Acesso em: 21/08/2008

BIO: Revista Brasileira de Saneamento e Meio Ambiente. Água: o ouro azul do século XXI. Rio de Janeiro, RJ, v.11, n. 21, jan./mar. 2002

BIO: Revista Brasileira de Saneamento e Meio ambiente. Centronetwork. Rio de Janeiro, $n^{\circ}$ 21. Ano XI. 2002. p.50

BRAGA, BENEDITO et al. Introdução à Engenharia Ambiental O desafio do desenvolvimento sustentável. 2ª edição. São Paulo: Pearson Prentice Hall, 2005.

CAUBET, Christian Guy. A água doce nas relações internacionais, ano 2006.

FIORI, Simone. Avaliação Qualitativa E Quantitativa Do Potencial De Reuso De Água Cinza Em Edifícios Residenciais Multifamiliares. Dissertação de Mestrado da Universidade de Passo Fundo (RS), 2005. Disponível: <http//www.usp.br>. Acesso em 12/11/2016.

GONÇALVES, R. F. (Coord.). Conservação de água e energia em sistemas prediais e públicos de abastecimento de água. Rio de Janeiro: ABES, 2009

HESPANHOL, I. Potencial de Reúso de Água no Brasil: Agricultura, Indústria, Municípios, Recarga de Aquíferos. Recursos Hídricos: APRH, Portugal, v. 23, n.2, p.43-65, nov. 2002.

LIBÂNIO, M. Fundamentos de qualidade e tratamento de água, 3eedição Campinas: Átomo, 2010.

MARIANE, Aline -Construtoras adotam sistemas de reuso e aproveitamento de água de chuva, além de métodos de construção seca, para contornar falta de água nas obras. Disponível em: http://construcaomercado.pini.com.br/negocios-incorporacaoconstrucao/161/artigo333851-1.aspx >Dez,2014. Acesso em: 12 Dez.2016. 
MAY, Simone. Conservação e Reúso de Água em Edifícios: Reúso De Águas Cinzas e Aproveitamento De Águas Pluviais Para Consumo Não Potável. Escola Politécnica da Universidade de São Paulo (USP),2009.

MAYOK, SIMONE. Caracterização, tratamento e reuso de águas cinzas e aproveitamento de águas pluviais em edificações. São Paulo, junho, 2009.

MIERZWA, J.C; VERAS,L.R; SILVA,M.C. Avaliação do Desempenho de Membrana de Ultrafiltração para Tratamento de Água Potável. VIII Seminário IberoAmericano. São Paulo: 2005.

OLIVEIRA, N. M.; SILVA, M. P. da.; CARNEIRO, V. A. Reúso da água: um novo paradigma de sustentabilidade. Revista Gestão e Desenvolvimento em ContextoGEDECON Edição Especial - Porangatu, v.2, n.1, p.146-157, jan. /jul. 2013.

OLIVEIRA, Sulayre Mengotti de. Aproveitamento da água da chuva e reúso de água em residências unifamiliares: estudo de caso em Palhoça - SC, junho de 2005.

OPAS (2001) Água e Saúde.Relatório.www.opas.org.br/sistema/fotos/agua.pdf. $<$ Acessado em abril de 2017>

PALHOÇA, Prefeitura Municipal de Palhoça. Disponível em: http://palhoça.sc.gov.br, acessado em novembro de 2004.

PROSAB, Uso Racional da Água em Edificações-Ricardo Franci Gonçalves (Coord.). Rio de Janeiro : ABES, 2006

SETTI, A. A. A Necessidade do Uso Sustentável dos Recursos Hídricos. IBAMA, Brasília, 1994.

SICKERMANN, Jack M. Gerenciamento Sustentável das Águas de Chuva: imprescindível para o futuro das grandes cidades do Brasil. Piauí, 2005. 9p. 
SILVEIRA, Bruna Quick da. REUSO DA ÁGUA PLUVIAL EM EDIFICAÇÕES RESIDENCIAIS, janeiro de 2008.

TOMAZ, P. Economia de água para empresas e residências: um estudo atualizado sobre o uso racional da água. $2^{\circ}$ Edição. São Paulo: Navegar Editora, 2005.

VESENTINI, J. W. Brasil, sociedade e espaço. 7. ed. São Paulo: Ática, 1999.

ZAMPIERON, Sônia Lúcia Modesto; VIEIRA, João Luiz de Abreu. "Poluição da Água”. Disponível em: <http://educar.sc.usp.br/biologia/textos/m_a_txt5.html> Inviato: Novembre, 2019.

Approvato: gennaio 2020. 